\title{
Misreadings of Static Energy Meters due to Conducted EMI caused by Fast Changing Current
}

\author{
Bas ten Have ${ }^{1}$, Tom Hartman ${ }^{1}$, Niek Moonen ${ }^{1}$, Frank Leferink ${ }^{1,2}$ \\ ${ }^{1}$ University of Twente, Enschede, The Netherlands, bas.tenhave@utwente.nl \\ ${ }^{2}$ THALES Nederland B.V., Hengelo, The Netherlands
}

\begin{abstract}
Readings of static energy meters can be affected by conducted electromagnetic interference (EMI). Previous research reported many cases where lower and higher readings of static energy meters were observed. In this paper experiments with a water pump, controlled by speed regulators, resulted in huge errors in energy readings of static meters with respect to a reference meter. The speed regulators are intended to be used in conjunction with such water pumps. The tests were performed using a non-distorted mains power supply created by a fourquadrant amplifier with an internal impedance in accordance with the standards. The deviations observed are between $\mathbf{- 9 1 \%}$ and $+\mathbf{1 7 5 \%}$ compared to the reference meter. The current waveforms attributed to these large deviations show large spikes with rise times of a few microseconds.
\end{abstract}

\section{INTRODUCTION}

Energy consumption in modern household situations is measured using static energy meters [1], [2]. For smart meters a communication link is added, with the purpose of using (price) dynamics in future smart grids. In previous research it was shown that conducted EMI can result in faulty static meters readings. In photo voltaic (PV) systems high interference levels were caused by either the power drives of fans [3] or active infeed converters connected to PV systems [4], [5]. These interference levels resulted in lower readings of static meters. When using pulsed currents, faulty energy readings were reported. Controlled experiments show that when static meters are loaded with a dimmer, a series of compact fluorescent lightning (CFL) and light emitting diode (LED) lamps, deviations between $-46 \%$ and $+276 \%$ [6], and $-32 \%$ and $+582 \%$ [7], [8], can arise. Using a standard commercially available water pump, energy deviations between $-61 \%$ and $+2675 \%$ were observed and reported in [9]. Furthermore, [9] shows that the deviations of the static meters depend on the impedance of the power supply. In the starting situation a standardized mains impedance was used, where lowering the impedance resulted in higher deviations, due to the capacitive behavior of the system which allows the high-frequency currents drawn by the load to flow easily. Changing to a higher impedance resulted in lower deviations. The measurements done with a buildings mains supply showed even worse results than using an ideal power supply with a low impedance. Higher energy readings were also observed by a consumer when using a standard commercially available water pump. The consumer complained about high energy meter readings, and experiments at the consumer's premises showed a large deviation of the installed smart meter compared to the reference meter.
In this paper lab experiments are described to elaborate on these findings. The experiments use three different power regulators intended to be used with a water pump, three different regulators are used, because it was measured that they gave a slightly different current waveform. The experiments are performed using an ideal power supply with a standardized mains impedance. The purpose of this paper is to present the results of the investigation of static meter deviations due to fast rising current spikes. The slope of these spikes is expressed and the steepness of the slope is correlated with the misreadings of the meters.

This paper is organized as follows: Section II describes the static meter measurement setup that is used and Section III shows the results of the measurements performed with the static meter setup, meter deviations, attributed waveforms and corresponding slopes. In Section IV the results of the measurements are discussed and a relation between the meter deviations and the steepness of the slopes is shown. Finally, in Section $\mathrm{V}$ it is concluded that fast changing currents contribute to high deviations of static meters.

\section{Measurement Setup}

The measurement setup consists of a Pacific Power Smart Source 140-TMX AC power source, which is a 4-quadrant amplifier, this generates an ideal, non-distorted, single phase sinusoidal voltage. To make sure that the tested static meters and load are not disturbed by the interference of a generic and uncontrolled mains supply network. A stable impedance is created using a line impedance stabilization network (LISN). This is connected to a series of ten static energy meters, which can be seen in Fig. 1. Meters that use different types of current sensors are included in the test setup: shunt resistor, current transformer, Hall effect-based current sensor and Rogowski coil. These represent the installed base of energy meters in the Netherlands and include new and old models. The energy meter readings of the static meters is corrected with the consumed energy of its following static meters. A water pump is used as a load and is placed inside a circular container, which is filled with water. The power drawn by the water pump is controlled using three different power, or speed, regulators intended to be used with it. These power regulators do not have fixed levels, therefore the power is tuned to three different levels per regulator: low (meaning: low power level, so low speed), medium and high. Also a $800 \mathrm{~W}$ heater combined with a regulator is used as load, to show the behavior with a resistive 
load. The method described in [10] is used to monitor the measured power consumption as indicated by the static meters. This method allows much faster evaluation of deviations as the time-difference of the blinking LED of the static meters is measured. This LED gives a much more accurate result than the standard display. As a reference for the static meters a Yokogawa WT500 power analyzer is used. It has a basic power accuracy of $0.1 \%$ and a sampling rate of $100 \mathrm{kS} / \mathrm{s}$, which should be adequate for reference as we are focused on EMI, and not on metrology. The current and voltage waveform drawn by the load are measured using a Pico Technology TA189 current probe and a Pico Technology TA043 differential voltage probe, these are connected to a Pico Technology Picoscope 4824, which is a PC based oscilloscope. This current probe has a $0.5 \mathrm{~dB}$ bandwidth of $100 \mathrm{kHz}$, while rise times of around $1 \mu \mathrm{s}$ will be measured during the experiments. Therefore, a closeup of a current spike as observed during the measurements is compared with a Tektronix AM 503 current probe, which has a bandwidth of $50 \mathrm{MHz}$. From Fig. 2, it can be seen that both probes show similar behavior. The complete schematic of the measurement setup is shown in Fig. 3.

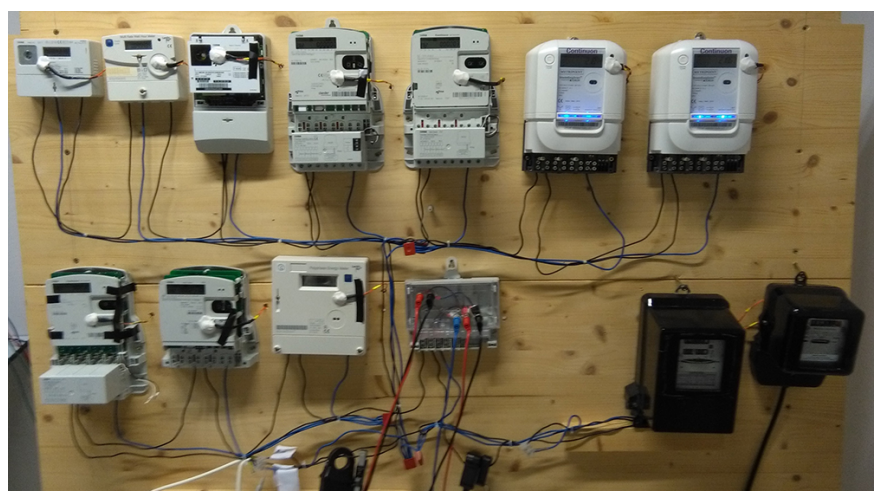

Fig. 1: Ten static meters placed in series.

TABLE I: Rise time, rising edge and slope of current spikes, load indicates water pump (WP), heater (HE) and regulator (Reg).

\begin{tabular}{|l|r|r|r|}
\hline Load & Rise time $[\mu \mathrm{s}]$ & Rising edge $[\mathrm{A}]$ & Slope $[\mathrm{A} / \mu \mathrm{s}]$ \\
\hline WP Reg 1 Low & 1.5 & 16.0 & 10.7 \\
\hline WP Reg 1 Medium & 1.9 & 15.2 & 8.2 \\
\hline WP Reg 1 High & 2.1 & 4.2 & 2.0 \\
\hline WP Reg 2 Low & 1.1 & 12.2 & 11.6 \\
\hline WP Reg 2 Medium & 2.3 & 16.2 & 7.1 \\
\hline WP Reg 2 High & 2.8 & 3.9 & 1.4 \\
\hline WP Reg 3 Low & 1.5 & 15.0 & 10.3 \\
\hline WP Reg 3 Medium & 1.6 & 14.8 & 9.2 \\
\hline WP Reg 3 High & 2.0 & 12.6 & 6.4 \\
\hline HE Reg 1 Low & 1.5 & 5.7 & 3.8 \\
\hline HE Reg 1 Medium & 1.5 & 5.3 & 3.7 \\
\hline HE Reg 1 High & 1.6 & 1.7 & 1.1 \\
\hline
\end{tabular}

\section{RESUlts}

The measurements are performed as described in the previous section. The deviations of the static meters, which is the

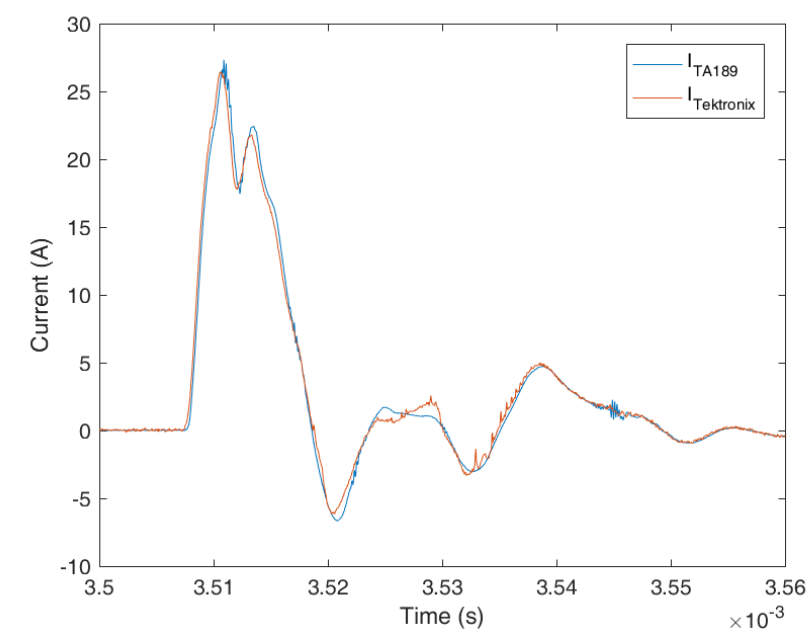

Fig. 2: Comparision between TA189 and Tektronix current probe, for a close-up of the line current as observed during the measurements.

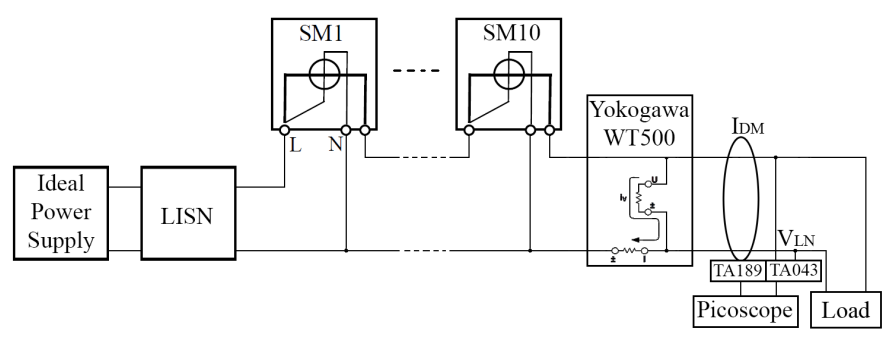

Fig. 3: Schematic overview of the measurement setup.

actual difference with respect to the reference divided by the reference value, are shown in Fig. 4 when they are loaded with the regulators and either the water pump or the heater. It is worthwhile to note that deviations of $-91 \%$ to $+10 \%,-19 \%$ to $+175 \%$, $-90 \%$ to $+16 \%$, and $-1 \%$ to $+4 \%$ are observed for regulator 1, 2 and 3 with water pump and regulator 1 with the heater, respectively.

In Fig. 5 the measured waveforms during the experiments are plotted. It shows the voltage waveform that is generated using the power supply without a load attached and the current waveforms generated in different load configurations. The water pump alone results in no deviations of the static meter readings. Fig. 5d shows waveforms obtained using a regulator combined with a heater, this shows how the regulator dims the load. In this situation no deviations of the static meters are observed, Fig. 4. This means that the specific combination of the water pump with the regulators causes the misreadings. The characteristics of the current spikes are shown in Table I, showing the rise time needed to rise from $10 \%$ to $90 \%$ of the peak value, which is used as a measure for the rise time in electronics for fast rise times [11]. Furthermore, the corresponding rising edges of the current peaks, and the related slopes are shown. Since the waveforms show multiple spikes, the values in Table I indicate 


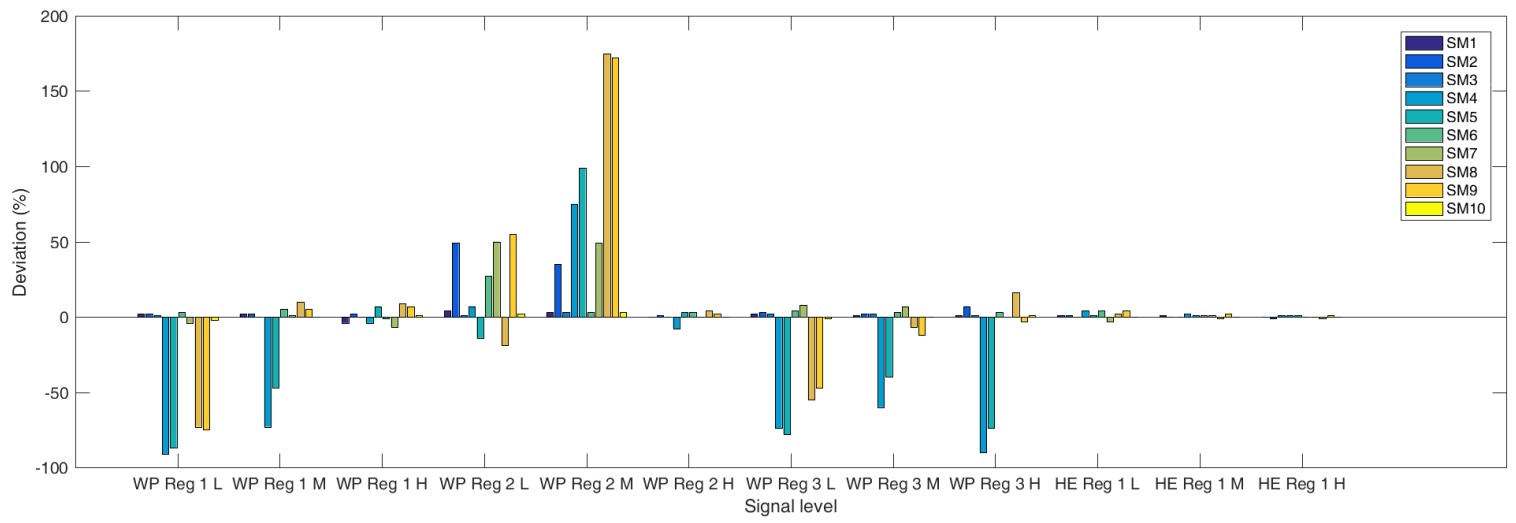

Fig. 4: Deviations [\%] between the 10 static meters (SM) and reference, for different combinations of water pump (WP), heater (HE) and regulator (Reg).

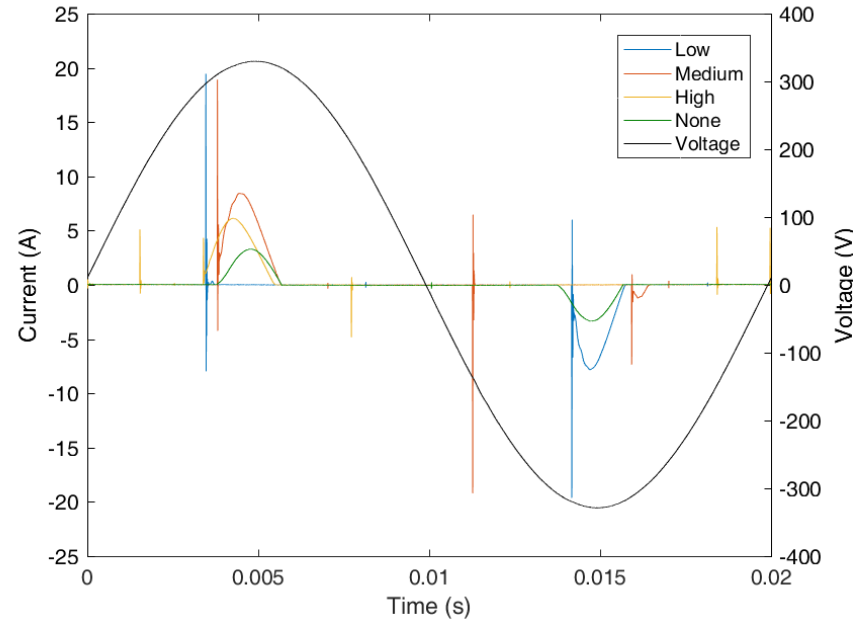

(a) Water pump with regulator 1 .

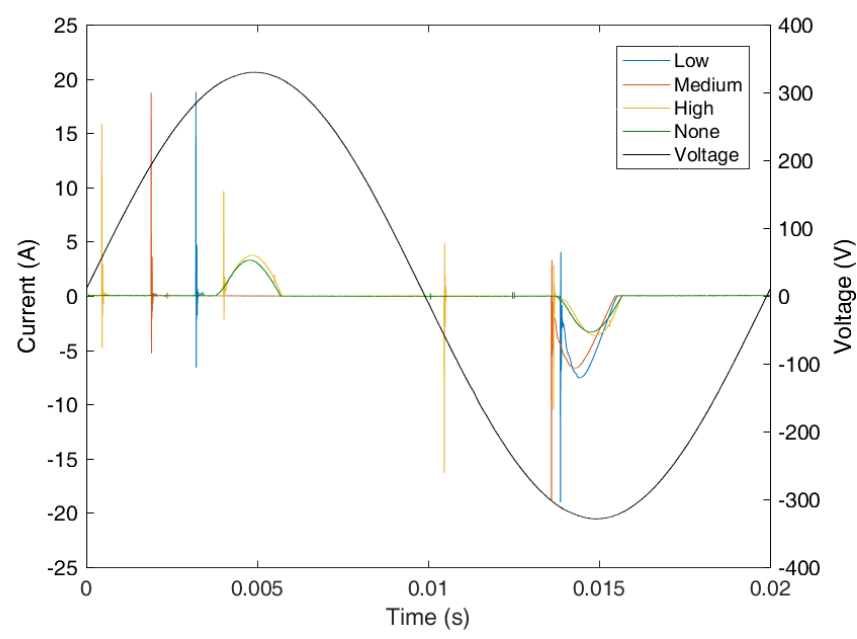

(c) Water pump with regulator 3 .

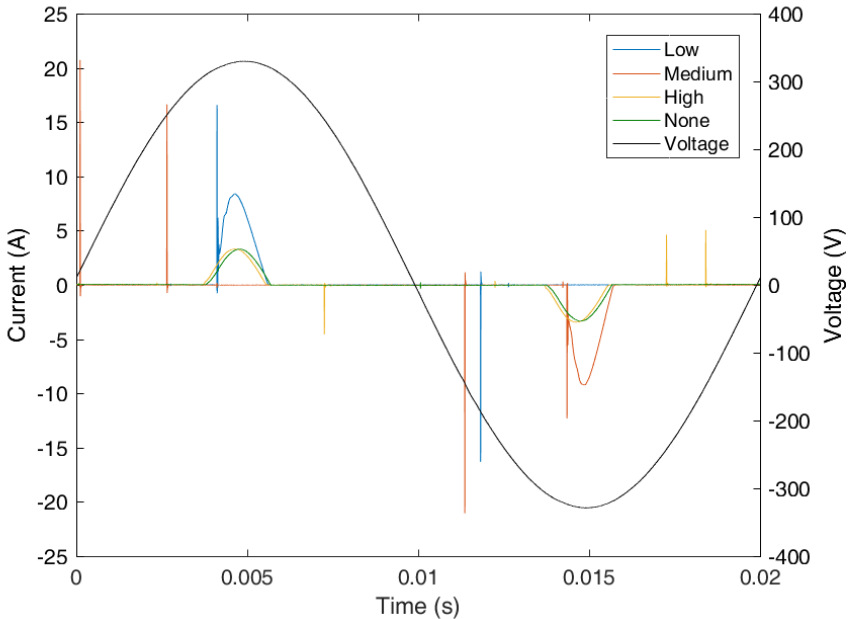

(b) Water pump with regulator 2 .

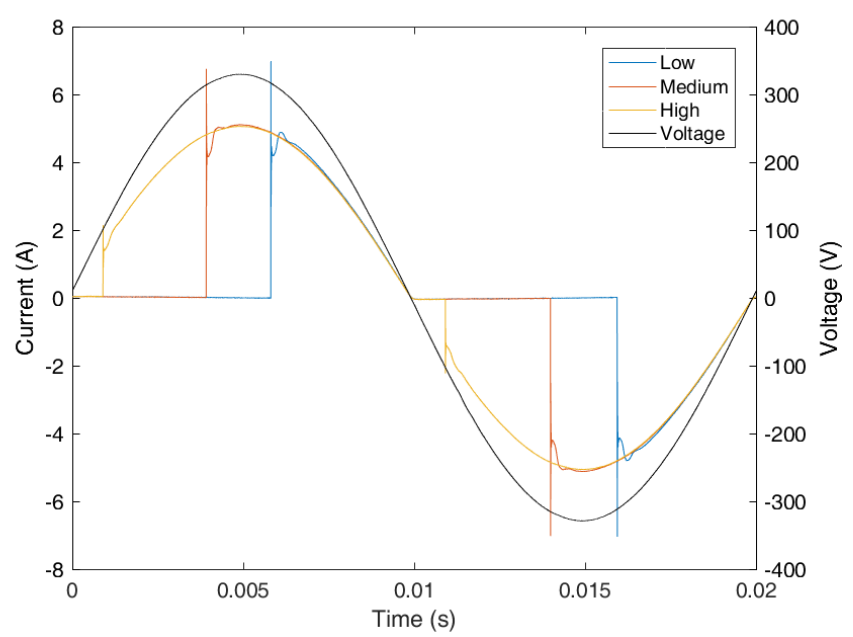

(d) Heater with regulator 1 .

Fig. 5: Current waveforms generated during measurements for different loads, the voltage is the wave generated by the power supply without a load. 
the maximum slopes found for a particular power level.

\section{DISCUSSION}

Several deviations of the static meters are observed during the experiments with the water pump and regulators. Also a correlation between the slope of the current spike and deviations can be seen, when looking at Table I and Fig. 4. When regulator 1 is used to create a low power level, the slope of the spike is $11 \mathrm{~A} / \mu$ s and deviations between $-91 \%$ and $+3 \%$ are observed. If the power level is changed to the medium level, resulting in a slope of $8.2 \mathrm{~A} / \mu \mathrm{s}$, the deviations are between $-72 \%$ and $+10 \%$. When the power level is changed to high power, almost no deviations are observed. A similar effect can be seen for regulator 3, however in this case the deviation of static meter (SM) 4 is still very high in the case of the high power level. For regulator 2 the largest deviations can be seen for the medium power level, which can be explained by looking at Fig. 5b, which shows more current spikes in this situation compared to the low power level.

Another observation is that also negative deviations are observed, while in previous work mostly positive deviations were shown to exist. Mainly negative deviations occur when using regulator 1 and 3 , while the use of regulator 2 shows mostly positive deviations. A reason for these deviations could be the low sampling rate of the static meters, [8]. In this case lower readings are measured, which could mean that the large spikes are missed completely by the static meters, because of the fast rise time. While for the higher readings measured, the spikes could be overestimated.

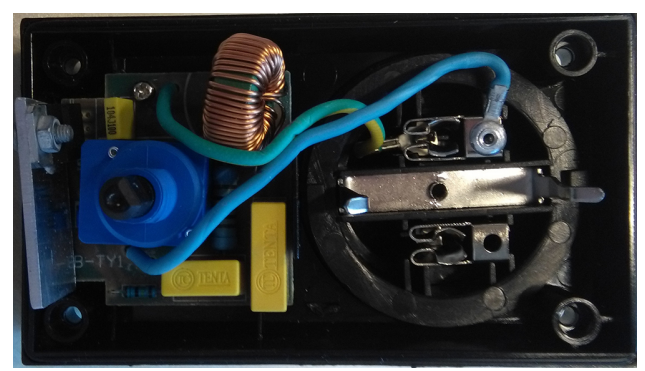

Fig. 6: Electronics inside the power regulator.

The working principle of the regulators, for a resistive load, is demonstrated in Fig. 5d. It can be seen that adjusting the power level of the regulator changes the phase where the current starts to flow. Due to the inductive behavior of the water pump, higher spikes occur in the current waveforms and the pulses occur either at the positive, negative or both sides of the sine wave, as shown in Fig 5a, Fig 5b and Fig 5c. After the measurements the regulators were opened to take a look at the electronic circuit used to regulate the power of the water pump, see Fig. 6. The circuit shows a triode for alternating current (TRIAC) which is controlled by a variable resistance. This variable resistance is the knob that is rotated by the user to regulate the power. One thing to note is that unless the inside of all the three regulators is exactly the same, the way the current is controlled differs from regulator to regulator. This could have to do with the cheap design of the regulators, the tolerances of the electrical components can result in a slightly different working conditions per regulator.

\section{CONCLUSION}

The measurement results show that a water pump, to be used in combination with a power regulator, can force static meters to give faulty readings. When an ideal power supply is used with a standardized mains impedance, deviations between $-91 \%$ and $+175 \%$ are observed. The waveforms show fast changing currents, with a high peak amplitude up to $16 \mathrm{~A}$ and a slope up to $11 \mathrm{~A} / \mu \mathrm{s}$. The deviations could be caused by the low sampling rate of the static meters, which could result in overestimating or completely missing the signal by the static meter. Another possibility is the large crest factor of the current waveform which could cause a short overload. The actual cause of misreadings, with focus on the effect of the slope of current spikes, is subject for further research.

\section{ACKNOWLEDGMENT}

This project has received funding from the EMPIR programme co-financed by the Participating States and from the European Union's Horizon 2020 research and innovation programme. The results found reflect the author's view only. EURAMET is not responsible for any use that may be made of the information it contains.

\section{REFERENCES}

[1] R. Quijano Cetina, A. J. Roscoe, and P. S. Wright, "Challenges for Smart Electricity Meters due to Dynamic Power Quality Conditions of the Grid: A Review," AMPS 2017 - IEEE International Workshop on Applied Measurements for Power Systems, Proceedings, 2017.

[2] R. Masnicki, "Some Remarks on the Accuracy of Energy Meters," 2018 IEEE International Conference on Environment and Electrical Engineering and 2018 IEEE Industrial and Commercial Power Systems Europe (EEEIC / I\&CPS Europe), 2018.

[3] F. Leferink, "Conducted interference, challenges and interference cases," IEEE Electromagnetic Compatibility Magazine, vol. 4, no. 1, pp. 78-85, 2015.

[4] J. Kirchhof and G. Klein, "EMV - Grenzwertlücke Wechselrichter stört Zähler," 24. Symposium Photovoltaische Solarenergy, Bad Staffelstein, 2009.

[5] J. Kirchhof, "Grenzwertlücke - Wechselrichter stört Elektrizitätszähler," EMV, Düsseldorf, 2010.

[6] F. Leferink, C. Keyer, and A. Melentjev, "Runaway energy meters due to conducted electromagnetic interference," IEEE International Symposium on Electromagnetic Compatibility, pp. 172-175, 2016.

[7] F. Leferink, C. Keyer, and A. Melentjev, "Static energy meter errors caused by conducted electromagnetic interference," IEEE Electromagnetic Compatibility Magazine, vol. 5, no. 4, pp. 49-55, 2016.

[8] C. Keyer and F. Leferink, "Conducted interference on smart meters," IEEE International Symposium on Electromagnetic Compatibility, pp. 608-611, 2017.

[9] B. Have, T. Hartman, N. Moonen, C. Keyer, and F. Leferink, "Faulty Readings of Static Energy Meters Caused by Conducted Electromagnetic Interference from a Water Pump," Submitted to Renewable Energy and Power Quality Journal (RE\&PQJ), 2019.

[10] B. Have, C. Keyer, and F. Leferink, "Monitoring of Power Measured by Static Energy Meters for Observing EMI Issues," 2018 International Symposium on Electromagnetic Compatibility (EMC Europe), pp. 903907, 2018.

[11] E. M. Chery and D. E. Hooper, "Amplifying Devices and Low-Pass Amplifier Design," IEEE Spectrum, vol. 6, no. 3, pp. 134-143, 1969. 